\title{
EL EXPERIMENTO DE LA RESONANCIA ELECTROMAGNETICA DE HERTZ DENTRO DE UNA PROPUESTA DE UN CURSO DE ELECTROMAGNETISMO
}

\author{
Pedro José Moreno Vargas* \\ José González Florez
}

\begin{abstract}
Having iike foundation the para digm of "Action at Distance" versus "Fields" and with the purpose of contributing fo the deveiopment of the Triad "Pedagogy-Teaching-Didactics, the present work has been eiaborated, as contribution to the proposal of a course of electromagnetism directed at the first levels university students.
\end{abstract}

\section{RESUMEN}

Teniendo como fundamento el paradigma de Acción a Distancia" versus "Campos" y con el fin de a portar al desarrollo de la Triada "Pedagogía-Enseñanza-Didáctica se ha elaborado el presente trabajo, como contribución a la propuesta de un curso de electromagnetismo dirigido a los primeros niveles universitarios.

\section{INTRODUCCIÓN}

Hasta antes del descubrimiento de Oersted, acerca de la influencia que ejerce una corriente eléctrica sobre un imán, faltaba mucha información y claridad sobre la relación entre estas dos entidades físicas. Y ya por esta época, Faraday, había incursionado en el terreno de las especulaciones investigativas alrededor de la posible relación, no sólo entre la electricidad y el magnetismo, sino de ellas dos, con la mayoría de fenómenos que estudiaba la filosofía natural. De ésa manera se puede considerar como uno de los pioneros en la realización de experimentos que apoyaran la teoría de la "Unificación de Campos".

Dentro de todas las investigaciones y trabajos desarrollados por Michael Faraday cabe señalar el resumen elaborado por el profesor Juan Carlos Orozco; el cual se reproduce en el cuadro $\mathrm{N}^{\circ} 1$.

\footnotetext{
${ }_{* *}^{*}$ Profesor Universidad Francisco de Paula Santander.

** Profesor Asociado, Departamento de Física. Universidad Pedagógica Nacional. 1996.
} 


\section{CONTRIBUCIONES CIENTIFICAS DE FARADAY EN LA FISICA ${ }^{1}$}

\begin{tabular}{|l|l|}
\hline AÑo & \multicolumn{1}{|c|}{ TRABAJOS } \\
\hline 1821 & Rotaciones electromagnéticas \\
1831 & Inducción electromagnética. Vibraciones acústicas. \\
1832 & Identidad de las electricidades provenientes de diversas fuentes. \\
1833 & Descomposiciones electrolíticas. \\
1835 & Descarga de electricidad a través de gases liberados. \\
1836 & Electrostática. Jaula de Faraday. \\
1845 & Relación entre luz, electricidad y magnetismo,: diamagnetismo; paramagnetismo. \\
1846 & "Pensamientos acerca de las vibraciones luminosas". \\
1849 & Gravedad y electricidad. \\
1857 & Tiempo y magnetismo. \\
1862 & Influencia de un campo magnético sobre las líneas espectrales del sodio. \\
& Líneas de fuerza y el concepto de campo. La energía de un imán reside fuera de \\
& su perímetro. La noción que luz magnetismo y electricidad estaban \\
interconectados.
\end{tabular}

\section{Cuadro No. 1}

En este artículo se resaltan los experimentos de la inducción electromagnética, la cual le permitió corroborar el vínculo entre la electricidad y el magnetismo junto con la existencia de las líneas de campo.

Otra preocupación del trabajo de Faraday era la medir el tiempo entre las interacciones de los fenómenos, es decir verificar que su propagación no se daba a distancia e instantáneamente como lo concebía la escuela Newtoniana de su época.

Fue Hertz quien más tarde involucrado por las propuestas de investigación, de la academia de Berlín, verifica que el tiempo transcurrido entre la emisión de las O.E.M. (Ondas Electromagnéticas) y la recepción de las mismas es medible.

Luego de varios años de exhaustiva labor -1886-1888- (García D. 1994), obtiene uno de sus primeros logros; construir un "Oscilador de Hertz". Aparato que iría a proporcionarle una de sus mayores satisfacciones. Demostrar la transmisión de la O.E.M. a través del espacio; las que posteriormente se constituirían en la piedra fundamental de la invención de la Radio, la modernización del telégrafo, la creación del teléfono y en general la columna vertebral de las comunicaciones de hoy.

Dada la velocidad del progreso de la ciencia y de la física en particular resulta imposible abarcar todos los temas de esta última en los cursos introductorios del nivel universitario; se propone en este trabajo organizar un curso que parta de la situación problemática que encuentra Faraday en el desarrollo del electromagnetismo, particularmente en la concepción del espacio, y la interacción entre cuerpos; culminan con los experimentos de $\mathrm{H}$. Hertz que permitieron darle fuerza a la teoría de campos.

\footnotetext{
${ }^{1}$ OROZCO, J.C. El encanto de la diferenciación. Tesis de Maestría en Docencia de la Física. Departamento de Física, Universidad Pedagógica Nacional. Santa Fe de Bogotá,
} 


\section{ANTECEDENTES}

Alrededor del primer tercio del siglo XVIII, con el experimento de Oersted se inicia el estudio del vínculo entre electricidad y magnetismo. Con este experimento, Faraday empezó a desarrollar una serie de ideas que lo llevaron a concebir una nueva visión del mundo con base en campos. (Abeles, F. y otros 1973).

François Arago, quien fuera uno de los primeros en enterarse del descubrimiento de Oersted, al poco tiempo logró demostrar la imanación del hierro dulce, lo cual dio origen al nacimiento del electroimán. Mas tarde, el propio Arago, quien viajó a París, dio cuenta del descubrimiento de Oersted a la Academia Francesa y en muy pocos días Ampére observó la interacción entre dos alambres que transportaban corrientes eléctricas.

En la mayoría de las investigaciones Ampére, que compartía las opiniones de Coulomb, - representantes de la escuela Newtoniana- proponía interacciones solo entre entes de la misma naturaleza, tales como la acción de una corriente eléctrica sobre otra o la acción de un imán sobre otro, motivo por el cual no dejó de sorprenderle el descubrimiento de Oersted. Con el ánimo de adentrarse en la compresión de tal fenómeno y al mismo tiempo de dar cuenta del mismo desde la teoría Newtoniana llegó a concebir a idea de que el magnetismo no fuera alguna sustancia distinta, sino sólo un aspecto de la electricidad. Dentro de sus hipótesis se tiene el suponer que los efectos magnéticos se podrían deber a corrientes dentro de los imanes, las cuales podrían interaccionar con las de otros imanes, obteniéndose así una explicación del descubrimiento de Oersted. De este problema surgió el descubrimiento de la atracción y repulsión de las corrientes eléctricas.

El hecho de que dos corrientes eléctricas paralelas se atraen cuando viajan en la misma dirección en tanto que se repelen cuando viajan en direcciones contrarias, dio pie para que propusiera un experimento parecido al de Oersted, en donde una de las corrientes permaneciera fija y la otra podía girar alrededor de la perpendicular a los ejes de ambos cables, comprobando que cuando las corrientes tenían direcciones opuestas, (caso repulsivo) el cable móvil daba un giro de 1800, hasta adquirir la posición contraría (atractiva), lo cual le permitió pensar que el experimento con el cable móvil podía ser análogo al de un imán que si originalmente se ubicaba en la dirección Norte-Sur, giraría $180^{\circ}$ por la acción de una corriente que fluyese en la dirección Este-Oeste ${ }^{2}$.

Por otro lado la teoría de Ampére implicaba una ligera desviación de la teoría de Newton, lo cual hacia en cierta forma, difícil la contrastación en el caso de las corrientes abiertas y además tropezó con un hecho contradictorio como fue el que dos corrientes paralelas que fluyen en la misma dirección se atraen y dos que fluyen en direcciones opuestas se repelen; lo cual contradecía la concepción Newtoniana dentro de la que se esperaba que en el primer caso las corrientes se repelieran, como sucede con dos cargas de igual clase; y en el segundo caso que se atrajesen lo mismo que dos cargas de clase diferente.

Es comprensible en parte la posición escéptica de Faraday hacia la teoría de Ampére, dado que esta estaba basada en la hipótesis de fluidos eléctricos, además que no proporcionaba una teoría unificada de la electricidad, que explicara efectos estáticos como

\footnotetext{
${ }^{2}$ BERKSON, Wuhan .,"Las teorías de los campos de fuerza desde Faraday hasta Einstein” Editorial Alianza, Madrid,1981, pp. 60-61.
} 
de corrientes abiertas. Por otra parte Faraday, al contrario de Ampére creía que esos fluidos eran más bien un estado especial de las fuerzas de un cuerpo cargado.

Estas inconsistencias que presentaban los resultados de Ampére a la luz de las concepciones newtonianas impulsaron a Faraday, entre otras a las investigaciones de las rotaciones eléctricas, la inducción electromagnética, las líneas de fuerza y el concepto de campo que le permitieron afincar la confianza en su teoría de campos. (Ver cuadro 1).

Posteriormente a Maxwell le correspondió escribir "la partitura de tan magistral pieza"; pero aún así, las investigaciones y la obra de Faraday parecía ser que carecía de más elementos, que le diera la relevancia sobre las demás teorías existentes de la época.

Años más tarde el científico Alemán $\mathrm{H}$. Hertz, impulsado por el reto planteado por la Academia de Berlín y la dirección de su profesor y amigo personal, el señor Vön Helmholtz, se dedicó a desarrollar una serie de investigaciones, a través de las cuales pudo mostrar experimentalmente la evidencia de la transmisión de las O.E.M. a través del espacio. Estos trabajos-precisamente vinieron a constituirse en una de las pruebas fehacientes de la teoría de campos de Faraday.

\section{LAS ONDAS HERTZIANAS}

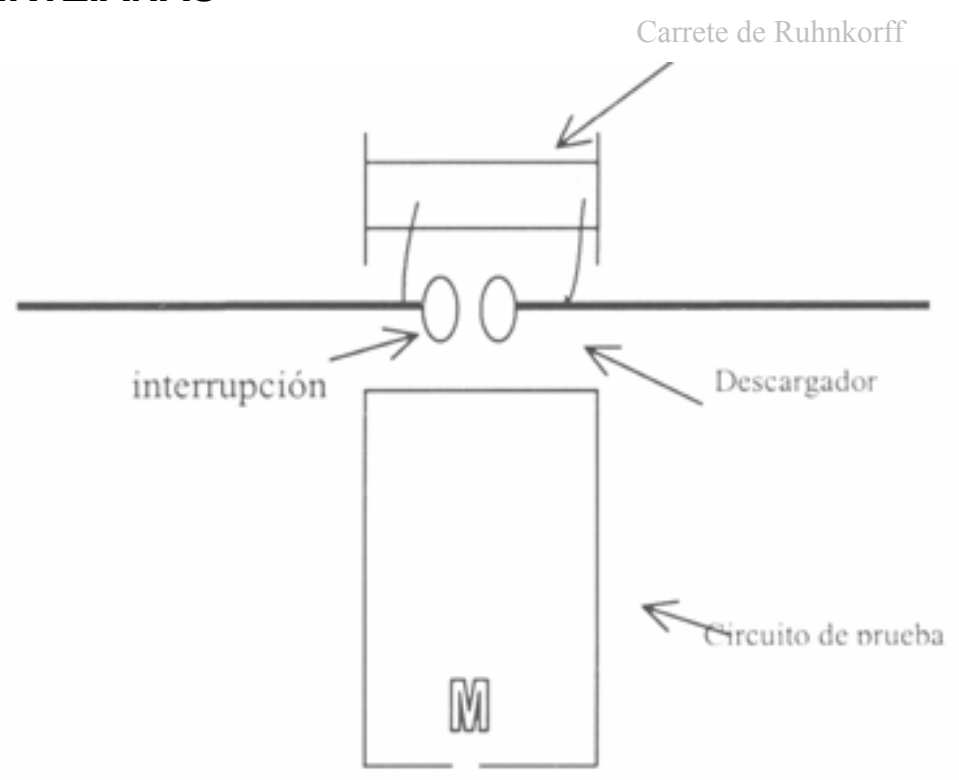

Heinrich R. Hertz, con el deseo de sacar adelante el desafió de la Academia enfocó sus investigaciones en una nueva dirección y como un primer paso se dio a la tarea de construir un Oscilador; el cual más tarde llevaría su nombre.

El Oscilador de Hertz, cuyo objetivo era la creación de O.E.M. muestra su actividad por medio de la emisión de chispas, gracias a un inductorio o carrete de Ruhnkorff, que al colocarlo en funcionamiento produce una serie de chispas en la corta interrupción que se halla en la mitad del conductor rectilíneo (figura 1), propicia la emisión de esa señal a su entorno. En ese salto de chispa se está dando un movimiento de corriente eléctrica de una forma alterna y consecutiva que se asemeja al cierre y apertura del circuito primario en el experimento de Faraday.

En el caso del experimento de Hertz, la presencia del salto de chispa no es otra cosa que la oscilación de una corriente entre los extremos de un circuito abierto, que permite la 
producción de una pequeña cantidad de la misma, entre los extremos de un microchispero (M) alojado en una espire testigo (circuito de prueba), ubicada en la cercanía de la primera. Aquí la manifestación, tanto de la oscilación en el circuito recto donde se genera la acción como en el circuito vecino o espire que se coloca como testigo, es visualizado en una serie de chispas que saltan de uno a otro extremo de la corta interrupción en el microchispero. Lo anterior está indicando que en la espira vecina se alcanza a generar una inductancia, que se manifiesta por medio de la pequeña chispa; pero que, en realidad, no es más que una débil corriente.

Según el desarrollo experimental llevado a cabo por Hertz, se podrían advertir los siguientes elementos similares en el proceso llevado a cabo por Faraday en su experimento de la inducción electromagnética, resumido en el cuadro No. 2.

\begin{tabular}{|l|l|}
\hline Inducción de Faraday & Oscilador de Hertz \\
\hline $\begin{array}{l}\text { inducción de una corriente en una espira } \\
\text { vecina por el movimiento de un imán ensu } \\
\text { cercanía. }\end{array}$ & $\begin{array}{l}\text { Inducción de una microchispa en una espira } \\
\text { testigo, colocada en la vecindad de circuito } \\
\text { abierto donde se genera una chispa. }\end{array}$ \\
\hline $\begin{array}{l}\text { Inducción por cierre y apertura, de un } \\
\text { circuito magnético, debido a un flujo de } \\
\text { corriente. }\end{array}$ & $\begin{array}{l}\text { Inducción de ondas electromagnéticas en } \\
\text { una espira testigo situada cerca del } \\
\text { oscilador de Hertz. }\end{array}$ \\
\hline $\begin{array}{l}\text { Inducción de una corriente en una espira, } \\
\text { debido al cierre y apertura de un circuito } \\
\text { primario provisto de una batería que se } \\
\text { halla en su vecindad. }\end{array}$ & $\begin{array}{l}\text { Inducción de una pequeña corriente, en una } \\
\text { espira testigo situada en la cercanía del } \\
\text { Oscilador de Hertz. }\end{array}$ \\
\hline
\end{tabular}

\section{Cuadro No. 2}

Como puede verse en la anterior sinopsis se presenta una gran similitud en los procesos llevados a cabo por Faraday en el desarrollo de su proceso de inducción, con los hechos que se dan durante el desarrollo experimental de Hertz, a lo largo del proceso de generación y recepción de las Ondas Electromagnéticas ${ }^{3}$.

En ambos procesos es fácil percibir la concepción de la transmisión de las acciones, pero a lo largo de acciones contiguas y que presuponen la duración de un tiempo en su consecución.

Además con base en ellos, pareciera decirnos Faraday: hay que dejar de lado la idea de que todo está totalmente constituido por diminutas partículas o átomos y que más bien es un continuo, como lo había afirmado Leibniz, en donde a manera de nudos de fuerza todo se encuentra lleno y permite la transmisión de esas fuerzas, tanto mecánicas como eléctricas y magnéticas e incluso dentro de aquello que se denomine vacío.

Los fenómenos, específicamente eléctricos y magnéticos, viajan a través del espacio y se dan o reproducen en posiciones distantes del punto de origen de su generación; si no con igual magnitud y eficiencia que en el punto de partida y en su cercan la, si, con un poco de menor vigor, pero de todas maneras con suficiente fortaleza como para dar evidencia clara de su generación y existencia. Por lo tanto, la teoría de Campos de Faraday se ha

\footnotetext{
${ }^{3}$ En un próximo articulo que se encuentra en prensa, se amplían los criterios que permiten comparar los desarrollos experimentales "Faraday-Hertz".
} 
venido a constituir en la piedra angular del electromagnetismo; y superando muchas controversias ha logrado ubicarse en el nivel de credibilidad que se merece.

Posteriormente, Hertz logra fortalecer los fundamentos de la teoría de campos, que permitiría avizorar un nuevo origen y una ruta más ágil y expedita a toda la tecnología de las comunicaciones e informática de que hoy gozamos; incluso de dar un invaluable viraje en las costumbres del entorno social y de la cultura en general de la Europa del feneciente siglo; avizorando al mismo tiempo unas mejores perspectivas para el siglo XX.

Al mirar detenidamente, los trabajos realizados, tanto por Faraday como por Hertz, alrededor de la inducción eléctrica y de las oscilaciones eléctricas respectivamente; se puede colegir claramente que son dos formas diferentes de ver y estudiar el mismo fenómeno. Puesto que, tanto Hertz como Faraday, llevan a cabo con sus trabajos la verificación de un proceso de Inducción electromagnética.

\section{A MANERA DE PROPUESTA}

Ante todo resulta interesante exponer lo que se considera en este trabajo como la triada Pedagogía-Enseñanza-Didáctica.

"La pedagogía es el área más filosófica de la práctica educativa; en ella son tematizadas, centralmente, las variables que tienen que ver con el sujeto: la cultura en la cual tiende a su realización como persona, las dimensiones psicológicas (por ejemplo, estilos cognitivos), las características económicas (la condición socioeconómica, la distancia casa-escuela), las condiciones sociológicas de su proveniencia (experiencia previa, que da lugar a los preconceptos, por ejemplo, del sujeto). No obstante, la reflexión pedagógica —valiéndose, como lo hace, de las llamadas "ciencias auxiliares de la educación" (antropología educativa, psicología educativa, sociología de la educación, etc.) es, mas bien, una reflexión "filosófica" sobre el sujeto, que un discurso estratégico sobre el aprendizaje.

La enseñanza la pensamos, más bien, en su carácter vinculado y vinculante con las disciplinas. Ella, como tal, tiene una estrecha relación con el saber y cuestiona: cuál, del acumulado disciplinar, ha de ser comunicado a los sujetos, en qué secuencie y con qué objetivos. La enseñanza, a su turno, depende de un "criterio epistemológico" que ha dado en llamarse "enseñabilidad"; como tal, este criterio dice relación a un proceso científico que tiende a socializar los resultados de los desarrollos investigativos; pero El mismo no esclarece cómo se puede realizar tal función en el aula o en la escuela. Por tanto, la transformación de la enseñabilidad en enseñanza equivale a la conversión del desarrollo de la socialización del conocimiento investigado disciplinarmente en tarea de formación de los sujetos en procesos sistemáticos y regulares de la escuela.

La didáctica, por su lado, la concebimos como el área aplicada tanto de la enseñanza como de la pedagogía. Ella tiende a establecer cómo hade ser comunicado un contenido, con qué indicadores de logro o verificadores del mismo; en suma, cuestione: cómo crear ambientes de aprendizaje. En este sentido, la didáctica es la concreción tanto de la pedagogía como de la enseñanza; con respecto a la primera "materializa" y "pone en escena" las "creencias del docente" (su "autoritarismo" o su "actitud democrática", etc.); 
con respecto a la enseñanza, hace explícita tanto su concepción como su conocimiento de la ciencia o de la disciplina en que se desempeña el docente" ${ }^{4}$.

Es de advertir que en este escrito se ha privilegiado la presentación del dominio del conocimiento (dentro de la Tríada, lo correspondiente a enseñanza).

La propuesta del curso parte de la situación problemática en la que se encontraba el electromagnetismo en la época de Faraday.

Partiendo desde la visión Newtoniana se presentan los trabajos de Ampére (interacción entre corrientes), se resaltan jas dificultades y anomalias que aparecen con estas explicaciones para así dar cabida a los trabajos de Faraday. En este momento surgen pregunta como: ¿la propagación de las acciones a través del espacio es realmente instantánea? Es decir, ¿La velocidad de propagación de la misma es infinita? Aquí es muy oportuno introducir el trabajo de Hertz como un apoyo experimental que permita dar respuesta a las preguntas formuladas.

\section{CONCLUSIONES}

Como se puede observar, la propuesta se construye sobre una de las preguntas que se hacen alrededor de las fuerzas de la naturaleza, particularmente las fuerzas electromagnéticas. La planeación del curso tic es pues sobre las teorías, leyes y productos ya dados, sino a través de interrogantes e inquietudes que se dan en el electromagnetismo.

Pensamos que de esta forma se pueden abordar temas contemporáneos sin necesidad de cubrir una serie de prerrequisitos que para lograrlos se requiere mucho tiempo.

Con ésta propuesta creemos que se está aportando a:

- Dar una visión del "quehacer" de la física.

- Enfatizar en el proceso y desarrollo del electromagnetismo.

- Comenzar, en este curso, a arañar los principios fundamentales del desarrollo de las comunicaciones.

- Al acercarnos a una revisión histórica es posible compenetrar a los estudiantes en los desarrollos investigativos de los creadores de las teorías.

- Al hacer un análisis y lograr una imagen inmediata de los contratiempos encontrados en estos procesos puede ayudar a fomentar el interés por la ciencia.

- En suma antes que encontrar respuestas a todo, se estimule a "Hacer las Preguntas, es decir se alimenta el espíritu científico.

Queda pues pendiente desarrollar el papel del alumno y del maestro para enmarcarlos, en esta propuesta, de una manera coherente en ésta propuesta. Esto es que el profesor

\footnotetext{
${ }^{4}$ VARGAS, G. y GONZÁLEZ, J. De la Informática Educativa a la Pedagogía Computacional. Articulo Universidad Pedagógica Nacional (en Imprenta).
} 
se asuma como un profesional de la educación y que el estudiante sea visto como una persona dinámica y colaboradora de un equipo de trabajo.

\section{BIBLIOGRAFÍA}

ABELĖS, F. ALLARD, G. y OTROS: (1993). Historia General de las Ciencias. Tomo III. La ciencia Contemporánea. Cap. IV. Electricidad y Magnetismo. Ediciones Destino. Barcelona. España.

ALONSO, Marcelo y FINN, Edward; (1976). Física Vol. II. Campos y Ondas. Cap. 16 y 17. Fondo Educativo Interamericano S.A. Impreso. E.U.A.

BAUTISTA, G., (1990). Consideraciones acerca del experimento en la Física. En: FISICA Y CULTURA Vol. $1 \mathrm{~N}^{0}$. 2. Bogotá Colombia.

BAUTISTA, G., (1997). Algunas consideraciones para la enseñanza del Electromagnetismo. En: FíSICA Y CULTURA. Vol. N ${ }^{0}$ 4. Bogotá Colombia.

BERKSON, William; (1981). Las teorías de los campos de fuerza Desde Faraday hasta Einstein. Alianza Editorial. Madrid España.

GARCÍA, Manuel y ROQUE, Xavier; (1990). Heinrich Hertz. Las ondas electromagnéticas. UABE. Colección Clásicos de la Ciencia Universidad Politécnica de Cataluña.

GARCÍA D. Manuel; (Enero, 1994).Heinrich Hertz. En: INVESTIGACIÓN Y CIENCIA. Barcelona España.

MULLIGAN, Joseph; (1994). Heinrich Rudolf Hertz (1857-1894). Colección de Artículos y direcciones. Garland Publishing, Inc. New York \&London.

OROZCO, Juan, (1996). El encanto de la diferenciación. Aproximaciones con Faraday a la Eneñanza de las Ciencias. Tesis de Grado. Departamento de Física U.P.N. Bogotá, 1996.

VARGAS, G. y GONZÁLEZ, J. De la Informática Educativa a la Pedagogía Computacional. Universidad Pedagógica Nacional. (Artículo en imprenta). 